\title{
Provision of adaptive guard band in elastic optical networks
}

\author{
Neclyeux S. Monteiro ${ }^{* *}$ (D), Alexandre C. Fontinele ${ }^{2}$, Divanilson R. Campelo ${ }^{2}$ and André Soares ${ }^{1}$
}

*Correspondence: neclyeuxsousa@gmail.com

1 Departamento de Computação, Universidade Federal do Piauí,

Teresina, Pl, Brazil

Full list of author information is

available at the end of the article

\begin{abstract}
Elastic optical networks are a network infrastructure capable of withstanding the high demand for data traffic from high-speed networks. One of the problems that must be solved to ensure the smooth functioning of the network is called Routing, Modulation Level and Spectrum Assignment (RMLSA). This work aims to propose a new approach to this problem with an algorithm to select the guard band in an adaptive way. Two algorithms for the adaptive selection of the guard band, called Guard Band according to Use of the Network (GBUN) and Guard Band by OSNR Margin (GBOM), are presented. The GBUN algorithm performs the guard band selection based on the usage level of network. On the other hand the GBOM algorithm uses an Optical Signal to Noise Ratio (OSNR) margin for the selection of the guard band. The performances of the proposed algorithms are compared with algorithms that use fixed guard band values and the adaptive proposal AGBA. The results showed that the GBOM algorithm presented a better performance in terms of bandwidth blocking probability for the studied scenarios. In general, GBOM also presents a better energy efficiency when compared to the other algorithms.
\end{abstract}

Keywords: RMLSA, Adaptive, Guard band, GBUN, GBOM

\section{Introduction}

Elastic Optical Networks (EONs), use light as a way of transmitting data, achieving high transmission rates with large amounts of data and low interference $[1,2]$. Such networks support multiple connections on a single optical fiber.

The use of the Orthogonal Frequency Division Multiplexing (OFDM) technology allows EONs to divide the optical spectrum into small frequency ranges, called slots. In this way, it is possible to attend the requests as different bandwidths, significantly improving the spectral efficiency of the optical layer [3].

In order to establish an optical circuit between two nodes in an OFDM optical network, a set of problems must be solved. One of these is the problem of Routing, Modulation Level and Spectrum Assignment, or an RMLSA problem for short [4]. The RMLSA problem consists of three steps: i) define a route for a pair of source and destination nodes; ii) select an appropriate modulation format for that route, and iii) choose a free spectrum range along the defined route to allocate the circuit.

(C) The Author(s). 2020 Open Access This article is licensed under a Creative Commons Attribution 4.0 International License which permits use, sharing, adaptation, distribution and reproduction in any medium or format, as long as you give appropriate credit to the original author(s) and the source, provide a link to the Creative Commons licence, and indicate if changes were made. The images or other third party material in this article are included in the article's Creative Commons licence, unless indicated otherwise in a credit line to the material. If material is not included in the article's Creative Commons licence and your intended use is not permitted by statutory regulation or exceeds the permitted use, you will need to obtain permission directly from the copyright holder. To view a copy of this licence, visit http://creativecommons.org/licenses/by/4.0/. 
In transparent elastic networks, data is transmitted from source to destination nodes as an optical signal; there is no conversion from the optical to the electronic domain in the nodes. When considering the imperfections of the physical layer (Impairment Aware IA), the circuits suffer degradations in the optical signal as they propagate from source to destination. These imperfections along the network can degrade the optical signal to such an extent that the Bit Error Rate (BER) becomes intolerable at the receiver node, making communication impossible [5].

In elastic optical networks, one or more free slots are used between the active circuits in order to reduce any interference between them. These free slots between the circuits are called Guard Bands (GB) [3]. The number of slots that will be used in the guard band affects the use of spectral resources directly. The larger the guard band, the better the Quality of Transmission (QoT) of circuits will be; however this increases the blocking probability due to the lack of spectral resources in the network. On the other hand, the smaller the guard band is, the worse the QoT will be and this will reduce the blocking probability due to the lack of spectral resources in the network [6].

This article presents an extension of the work presented in [7] were the algorithm called Guard Band according to Use of the Network (GBUN) was first presented. This algorithm takes into account the state of spectrum usage of the network to determine the guard band to be used for the establishment of a given circuit. Furthermore, this extended work also includes a new proposal, called Guard Band by OSNR Margin (GBOM), which uses an OSNR (Optical Signal to Noise Ratio) margin to determine the guard band that will be used by the new circuit.

An evaluation is presented comparing the proposals with fixed values found in the literature for the guard band and the adaptive proposal presented in [8]. The results show that selecting the guard band in an adaptive way is more efficient to reduce the probability of general blocking of the network. The scenarios evaluated and results presented are innovative in comparison to what was presented in [7].

The remaining sections of this article are organized as follows. In Section 2 the elastic optical network architecture is presented and the physical layer model adopted in this work is described. Section 3 presents the RMLSA problem and the guard band selection problem and discusses related work. The proposed algorithms are presented in Section 4. Section 5 presents the performance evaluation of the proposals. Finally, the conclusions of the work are highlighted in Section 6.

\section{Elastic optical network architecture and physical layer model}

The incorporation of OFDM technology in optical networks has allowed the emergence of a new type of optical transport network called an elastic optical network, as proposed in [9]. OFDM technology allows the optical spectrum to be divided into fine granularity, also called frequency slots. In an optical network, optical circuits have to be established for clients (nodes) to communicate with each other. In elastic optical networks, optical circuits are made up of one or more frequency slots. The number of frequency slots depends on the bandwidth required for each optical circuit. Figure 1 presents an example of dividing the optical spectrum into frequency slots.

In Fig. 1, white slots represent free slots and color slots represent circuit-occupied slots. The free slots between the slots occupied by circuits represent the guard bands. Guard bands are used to reduce interference between adjacent circuits [10]. Also illustrated in 


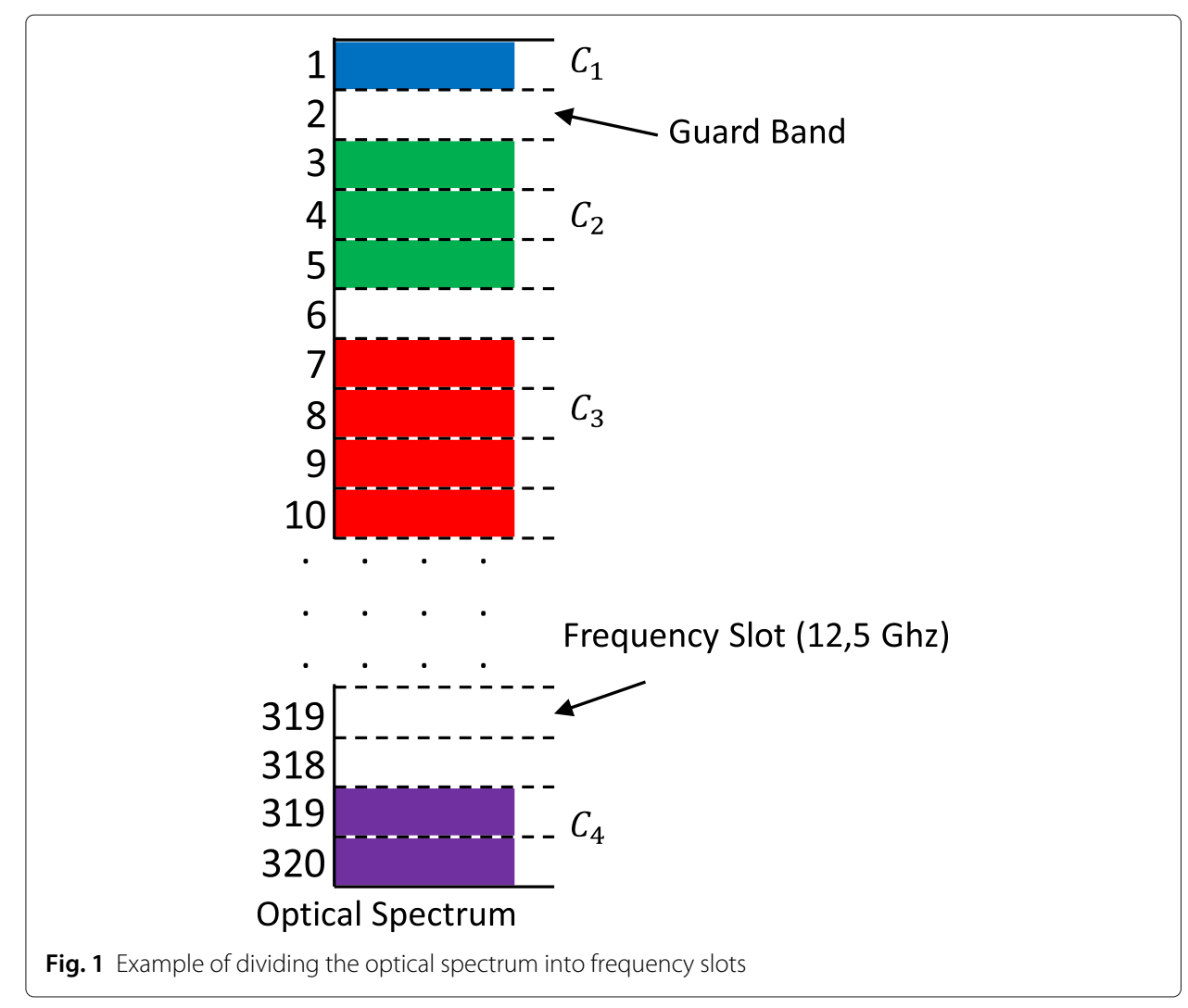

Fig. 1 is the allocation of four circuits with distinct bandwidths. Frequency slot allocation is adjusted to meet the required bandwidth. For example, the circuit $c_{1}$ allocates only one frequency slot, while the circuit $c_{4}$ allocates five frequency slots.

To guarantee flexibility in the use of the optical spectrum, elastic optical networks use two technologies: Bandwidth Variable Transponders (BVT) and Bandwidth Variable Wavelength Cross-Connects(BV-WXC). BVTs ensure flexibility in the use of spectral resources for the transmission and reception of data at source and destination nodes. BVTs are used to adjust the bandwidth according to the transmission bit rate or modulation format adopted. BV-WXCs enable optical switching of circuits using dynamically adjusted spectrum width circuits [3]. Figure 2 illustrates the setup of BVTs, BV-WXCs, amplifiers, and optical fibers in an elastic optical network.

As it can be seen in Fig. 2, the BVTs are located at the elastic network border nodes, allowing the transmission and reception of client data. After the process of admitting a request, the optical circuit is established from a BVT on the origin node. This circuit transparently traverses the BV-WXC at the intermediate nodes (transient signals), and ends at a BVT at the destination node.

The attenuation of the signal is greater the longer the distance it travels. Therefore there is a need to amplify the optical signal so that it can be detected at the destination. Amplifiers are used to compensate for optical signal power losses inserted by BV-WXCs and fibers. Optical amplification is usually performed by Erbium Doped Fiber Amplifiers (EDFA). At the time of amplification, these EDFAs introduce noise due to the Amplified Spontaneous Emission (ASE) [11]. 


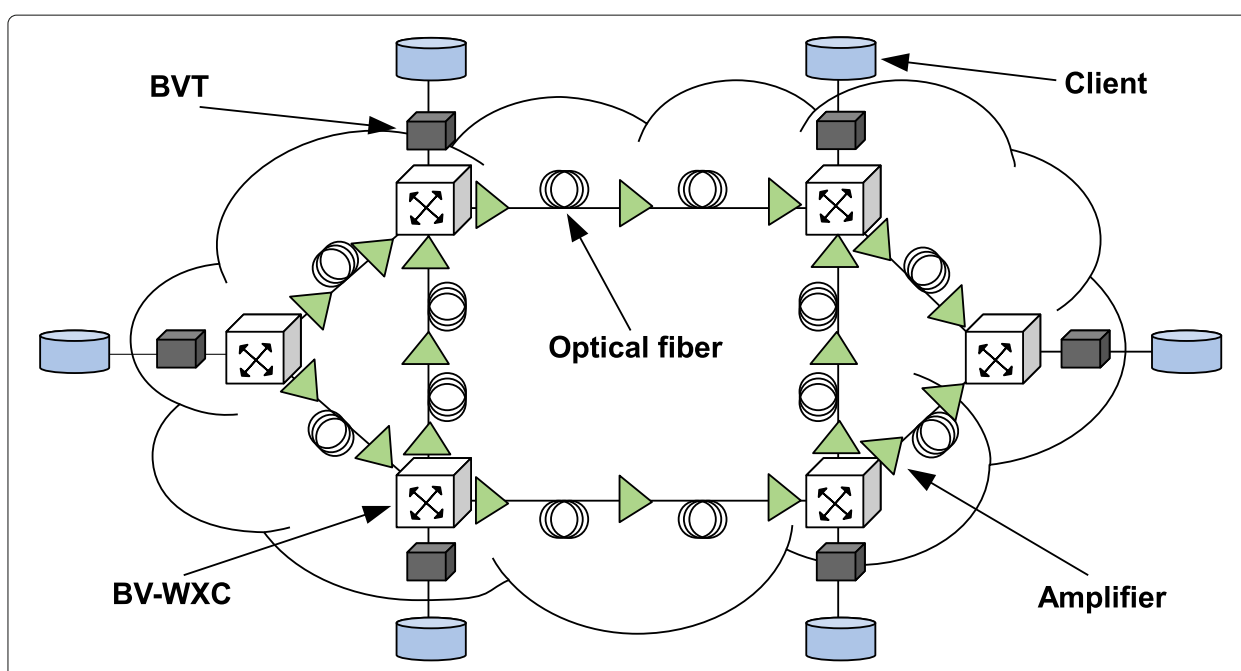

Fig. 2 Elastic Optical Network Architecture

In addition, noise generated by Nonlinear Impairments (NLIs) still occurs when the signal propagates through the fibers. Nonlinear effects include [11]: Self-Phase Modulation (SPM), Cross-Phase Modulation (XPM) and Four-Wave Mixing (FWM) [11].

In this work, we adopted the physical layer model presented in [12] and [13] to measure the impacts of ASE and NLIs on the Optical Signal to Noise Ratio (OSNR). The model assumes polarization-multiplexed multi-symbol modulation formats with coherent detection and dispersion compensation performed by digital signal processing in the electric domain on the receiver side. The OSNR calculation for a circuit $i$ using a route $r_{i}$ is expressed by:

$$
\operatorname{OSNR}_{i}=\frac{I}{I_{A S E}+I_{N L I}} .
$$

The variable $I$ is the signal's Power Spectral Density (PSD), $I=P_{T X} / \Delta_{f}$, where $P_{T X}$ is the power of the signal and $\Delta_{f}$ is the bandwidth of the circuit. The PSD of the ASE noise is given by:

$$
I_{A S E}=\sum_{l \in r_{i}} N_{l} I_{A S E}^{0}
$$

where $N_{l}$ is the number of link spans $l$ and $I_{A S E}^{0}=\left(G_{A M P}-1\right) F h v$. The variable $F$ is the Noise Figure (NF) of the amplifier [5], $h$ is Planck's constant, $v$ is the frequency of light and $G_{A M P}$ is the gain of the optical amplifier. The PSD of NLI is given by:

$$
I_{N L I}=\sum_{l \in r_{i}} N_{l} I_{N L I}^{l}
$$

where $I_{N L I}^{l}$, the PSD of NLI noise in a single span of link $l$, is expressed by the Eq. 4 [10]:

$$
\begin{gathered}
I_{N L I}^{l}=\varrho\left(\epsilon+\sum_{j} \ln \left[\frac{\left(\Delta_{f_{i j}}+\frac{B_{j}}{2}\right)}{\left(\Delta_{f_{i j}}-\frac{B_{j}}{2}\right)}\right]\right), \\
\varrho=\frac{3 \gamma^{2} I^{3}}{2 \pi \alpha\left|\beta_{2}\right|}, \\
\epsilon=\operatorname{arcsinh}\left(\frac{\pi^{2}\left|\beta_{2}\right|}{2 \alpha} B_{i}^{2}\right),
\end{gathered}
$$


where $j$ is another circuit using link $1, B i$ and $B j$ are, respectively, the bandwidths for circuits $i$ and $j, \Delta_{f_{i j}}$ is the center frequency spacing between circuits $i$ and $j, \gamma$ is the nonlinear fiber coefficient, $\beta_{2}$ is the fiber dispersion parameter and $\alpha$ is the power attenuation caused by the fiber.

For this work, the OSNR is used as the QoT criterion. If the OSNR value reached by the circuit is not above or equal to a pre-established OSNR threshold, then the circuit establishment may be blocked due to the absence of QoT. A QoT block can happen in two ways: i) inadequate QoT for the New circuit (QoTN) or ii) inadequate QoT for one or more circuits already active in the network (QoTO). QoTN is the blockage suffered if a new circuit does not reach adequate QoT levels. Even if a new circuit meets this requirement, it may still be blocked if the establishment of the new circuit affects the QoT of already established circuits, thus causing QoTO [14].

\section{RMLSA and the guard band selection problem}

To make a better use of spectral resources of networks, the RMLSA problem must be solved. In the first step of the problem it is necessary to select a route between the source and destination nodes. Figure 3, presents an example topology and illustrates the routing problem with source at node 1 and destination at node 4 .

Figure 3 shows that for a request originating at node 1 and destination at node 4 , there are 4 possible routes. These routes are: $1-2-3-4 ; 1-2-5-4 ; 1-6-5-4$ and $1-6-5-2-3-4$. The choice between them depends of the routing policy adopted.

After the routing stage, the next step of the problem is to choose the modulation format. The modulation format chosen together with the required bandwidth determines how many slots are needed to meet the request. Figure 4 shows some examples of modulation formats used by optical networks. Figure 4 indicates that the higher the spectral efficiency of the modulation, the smaller is the optical reach.

The modulations that have a higher spectral efficiency (like 32QAM), tend to suffer more noise from the physical layer; therefore, they have a lower spectral range. Consequently, the choice of the modulation format is related to the size of the chosen route. In this way, less spectrally efficient modulations are more adequate because they have a larger optical range (like BPSK) [15].

In the third and last part of the problem, it is necessary to choose a spectrum-free range to allocate the optical circuit. At this point, it is necessary to attend the restrictions

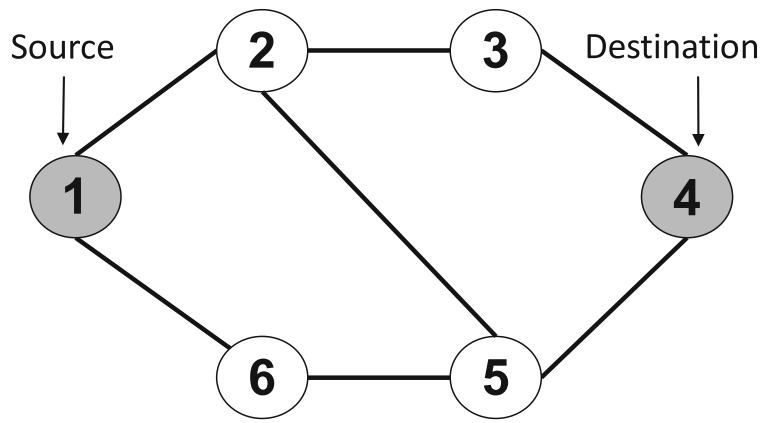

Fig. 3 Routing problem between nodes 1 and 4 


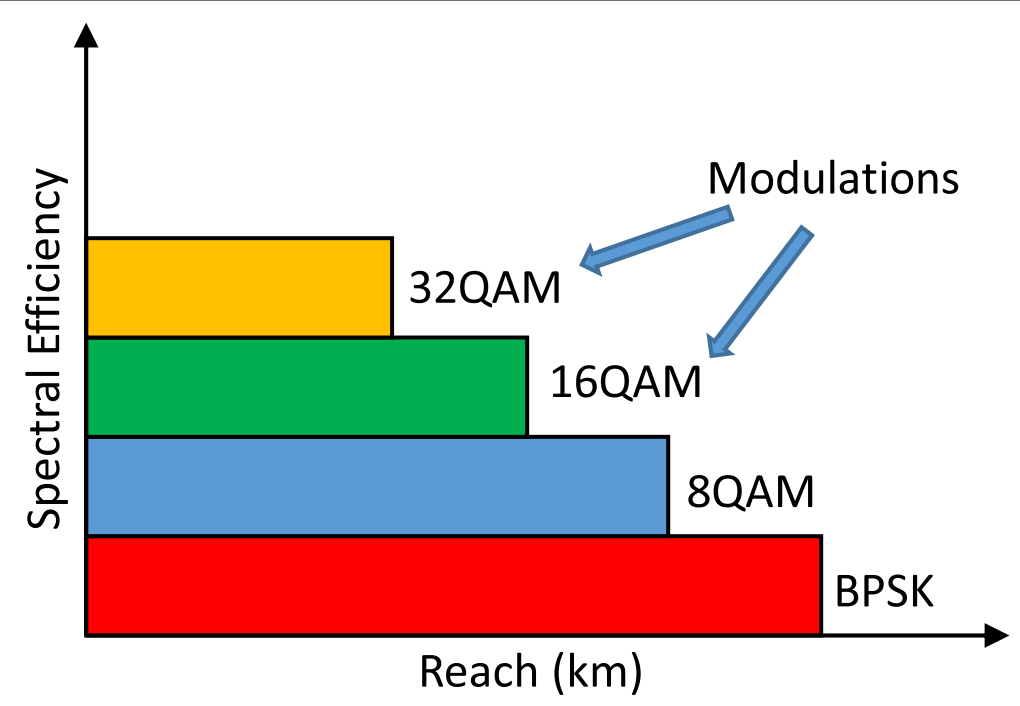

Fig. 4 Example of modulations used by elastic optical networks

of continuity and contiguity in order to establish the circuit. Figure 5 demonstrates the restrictions associated with spectral allocation.

In the continuity restriction, in order to avoid converting the optical signal from the optical domain to the electronic domain, it is necessary to keep the optical signal in the same spectral range in all the links between the source and destination nodes of the request. Therefore, the same range of the chosen slots set to allocate the request must be available in all the links of the chosen route. Figure 5 shows that it is possible to allocate a request that requires 2 slots (Request (b)) because the same spectrum range is free in all links of the route.

The contiguity restriction requires that the slots reserved for the establishment of the circuit are adjacent in the optical spectrum. In the contiguity constraint, for requests that require 2 or more slots, they must be allocated adjacent. In Fig. 5 there is a request that requires 3 slots (Request (a)) that cannot be attended because it does not contain 3 free and adjacent slots.

As circuits are established and disconnected, free gaps appear in the spectrum, thus increasing the probability that continuity and contiguity constraints will not be respected.

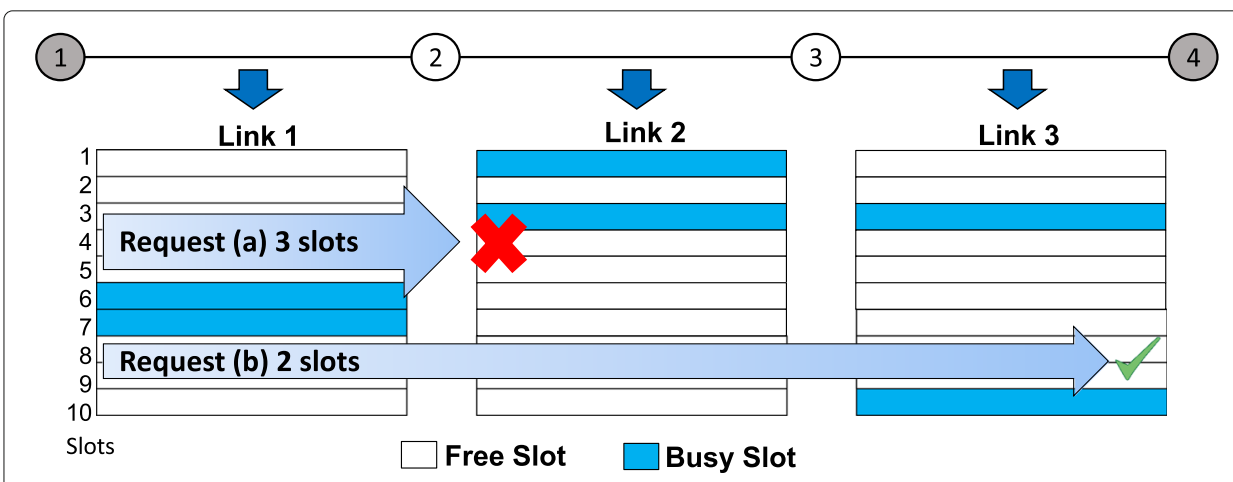

Fig. 5 Continuity and contiguity restrictions 
As a result, some requests will not be attended even if there are sufficient slots, causing Fragmentation (Frag) blocking. When the network does not have enough slots to attend the request, Absence of Free Spectrum (AFS) blocks it.

As previously reported, in elastic optical networks the guard band is used to reduce interference between the active and adjacent circuits in the optical spectrum. When searching for free slots to allocate the circuit, respecting the continuity and contiguity restrictions, the RMLSA algorithm must also respect the guard band between the circuits.

One should be careful in choosing the number of slots to be used as a guard band. Figure 6 shows the behavior of the bandwidth blocking probability as a function of the fixed number of slots selected to be used as the guard band. The scenarios used in the study presented in Fig. 6 are detailed in Section 5.

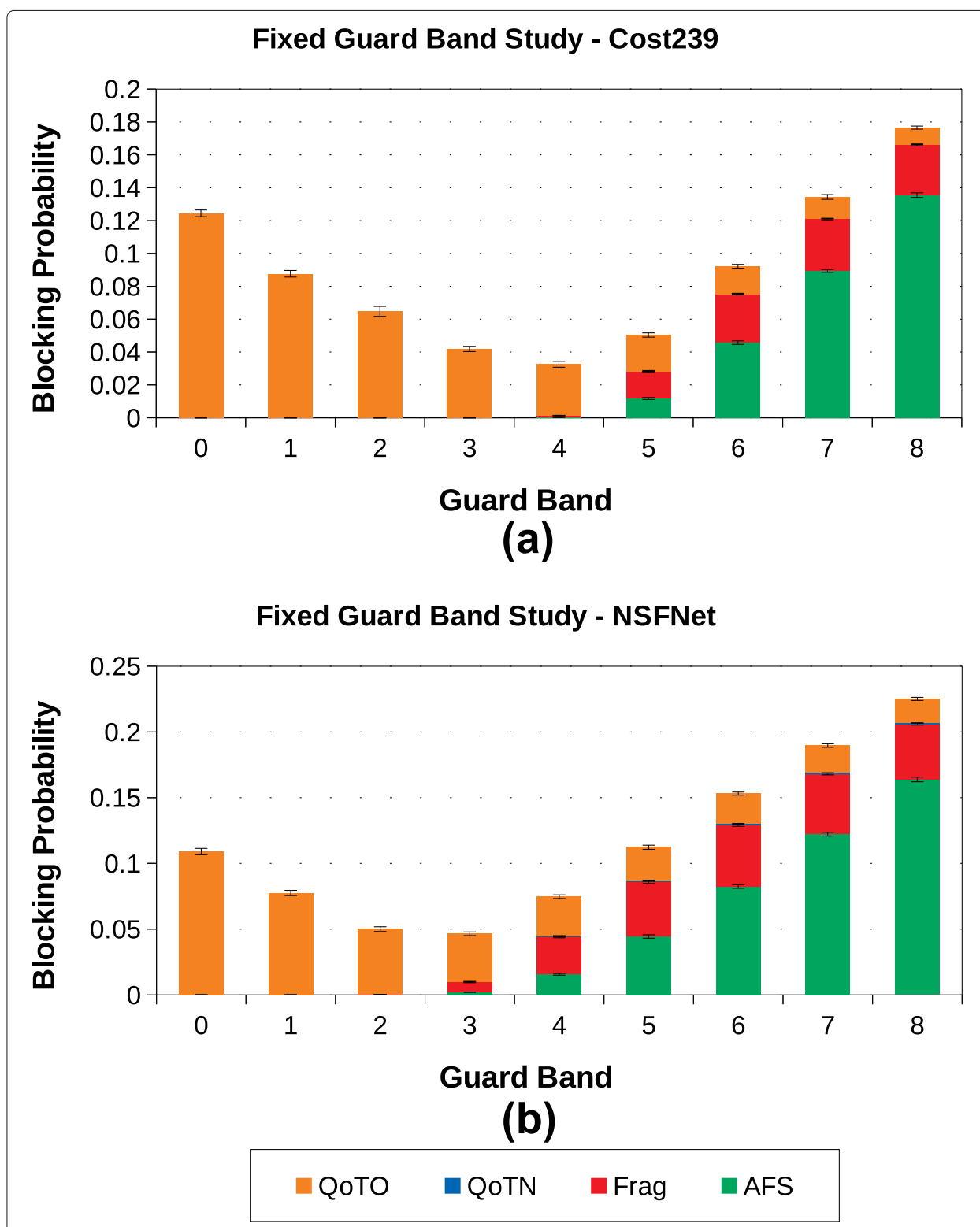

Fig. 6 Bandwidth blocking probability as a function of the guard band for $\mathbf{a}$ Cost239 and $\mathbf{b}$ NSFNet topologies 
As it can be seen in Fig. 6, as the guard band increases, the probability of blocking due to the Quality of Transmission of Other circuits already active in the network (QoTO) decreases. These blocks of QoTO-type circuit requests are connected to interference between the circuits. In contrast, the Fragmentation, AFS and QoTN blocking components tend to increase due to a lack of network spectral resources.

Looking at Fig. 6a, considering a load of 400 Erlangs, the 4-slots guard band presented a lower probability of blocking for the Cost239 topology. In the NSFNet topology, with a 200 Erlangs load, a lower blocking probability was obtained using a fixed guard band of 3-slots, as shown in Fig. 6b.

An insufficient guard band can prevent an optical circuit from reaching its destination due to the noise caused by the physical layer. However, an excessive assignment of the guard band may cause blockages due to spectral insufficiency. Therefore, optimizing the choice of the guard band is an important factor in planning elastic optical networks.

Some works found in the literature $[6,8,16]$ investigated the problem of adapting the guard band to perform a balance between blocking due to a lack of spectral resources and blocking due to the quality of the transmission.

The authors [16] performed a blocking probability study using singlepath and multipath routing. The authors also studied how different guard band sizes affected the performance of the routing algorithm. As in [16] there is no way to calculate the quality of transmission of the circuits and neither the interference between them, and a larger guard band leads to a higher blocking probability. Because of this, all blockages are generated by a lack of spectral resources.

In [8] an adaptive guard band selection algorithm called Adaptive Guard Band Assignment (AGBA) was proposed. The intention of the AGBA algorithm is to avoid overassigning the guard band and thus achieve a more efficient use of the optical spectrum. AGBA uses only two possible values for guard band assignment: 1 and 2 slots. These values are assigned according to the number of links of the chosen route. If the route chosen for a particular circuit has 4 or less links, the assigned guard band will equal to 1 slot. Otherwise, the guard band will be equal to 2 slots.

In [6] the authors conducted a study to identify the optimal guard band size to be fixed in order to reduce the impacts caused by non-linear effects. The authors presented a study similar to the one presented in Fig. 6. As the guard band increases, the QoT blockage decreases rapidly. However, the blockage due to the lack of available spectral resources increases. In this study, the authors identified that total network blocking is minimized with a $7 \mathrm{GHz}$ or $8 \mathrm{GHz}$ guard band.

\section{Proposed algorithms}

In this section, the two proposals of this work for the adaptive guard band selection are presented. The first proposal is based on the level of network spectrum utilization at the time of a circuit request. The algorithm is called Guard Band according to Use of the Network (GBUN). Figure 7 shows the application flowchart of the GBUN algorithm by the control plane to establish a circuit.

It is worth noting that the control plane comprises a set of protocols responsible for the dynamic provisioning of circuits [17]. In the case of this work the control plane is responsible for applying the routing, modulation selection and spectrum assignment algorithms. It also stores information about the network architecture and its current state. 


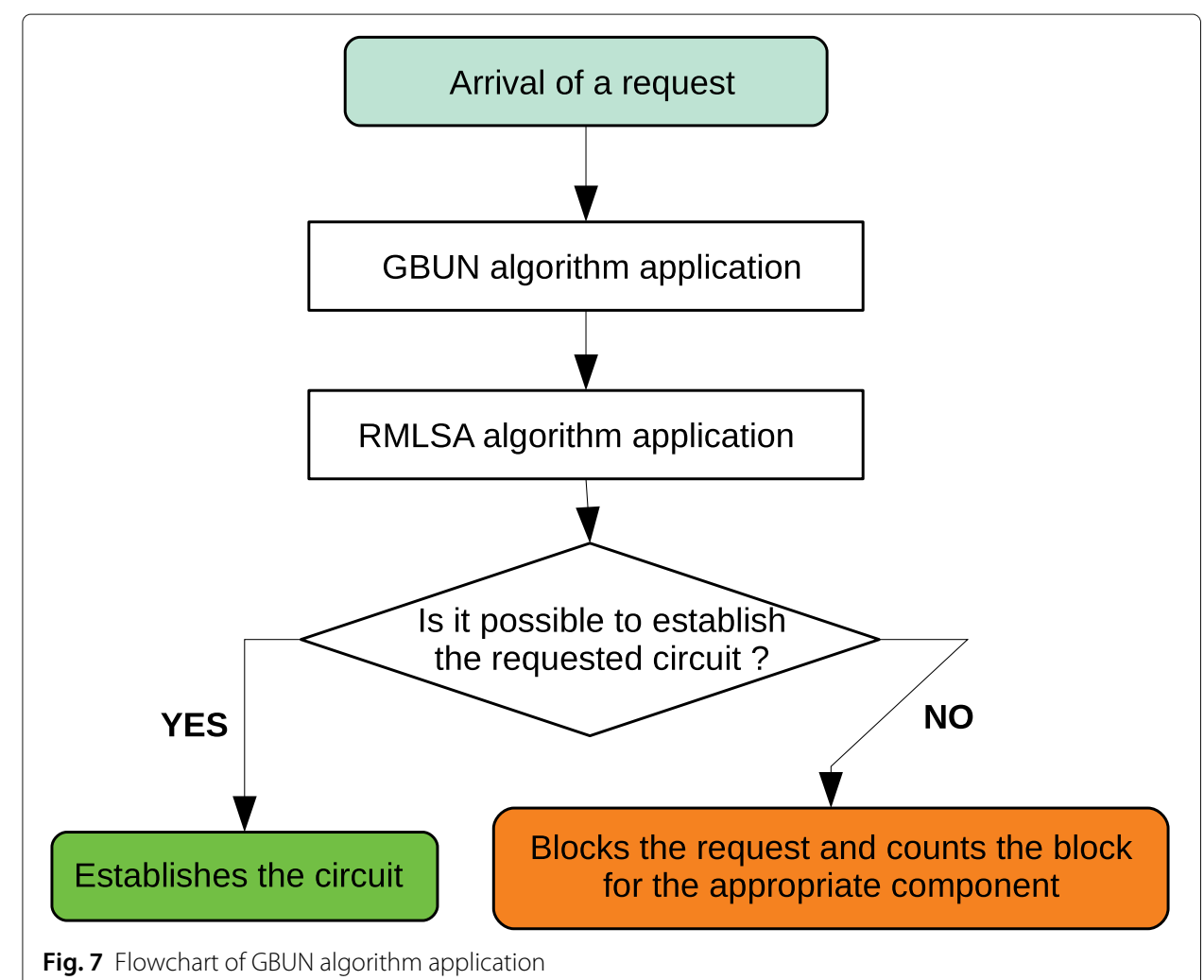

Figure 7 shows that the GBUN algorithm is applied immediately after the arrival of a circuit request. After the GBUN algorithm defines the guard band, the control plan applies the RMLSA algorithm selected by the network operator. Then, the control plan checks if it is possible to establish the new requested circuit. If it is not possible to establish the new circuit, the blocking is identified and accounted for by one of the blocking components already mentioned (Fragmentation, AFS, QoTN or QoTO). If it is possible to establish the new circuit, the control plan allocates the resources defined by the RMLSA algorithm.

Experiments were conducted with values for the guard band ranging from 0 (zero) slots to 8 slots. The experimental results showed that the guard band with 8 slots generated a higher blocking probability compared to the blocking probability generated by other guard bands (like 4 slots) for average loads (Like, 200 or 250 Erlangs) and high loads (Like, 350 or 400 Erlangs). These results can be seen in Fig. 6. However, when the guard band with 8 slots is used for low loads (like, 50 or 100 Erlangs) the blocking probability tends to be lower than the blocking probability generated by the guard band with 1 slot.

Due to this behavior, the GBUN algorithm aims to select a lower guard band value when the network is in high demand and a higher guard band value when the network is low demand. The spectral usage of a network link is calculated as follows:

$$
\text { Spectral usage }=\frac{\text { Number of used slots }}{\text { Total slots of the link }} .
$$

Therefore, network spectrum usage is defined by the sum of the spectral usage of all links divided by the number of links in the network. Figure 8 shows the spectrum usage 


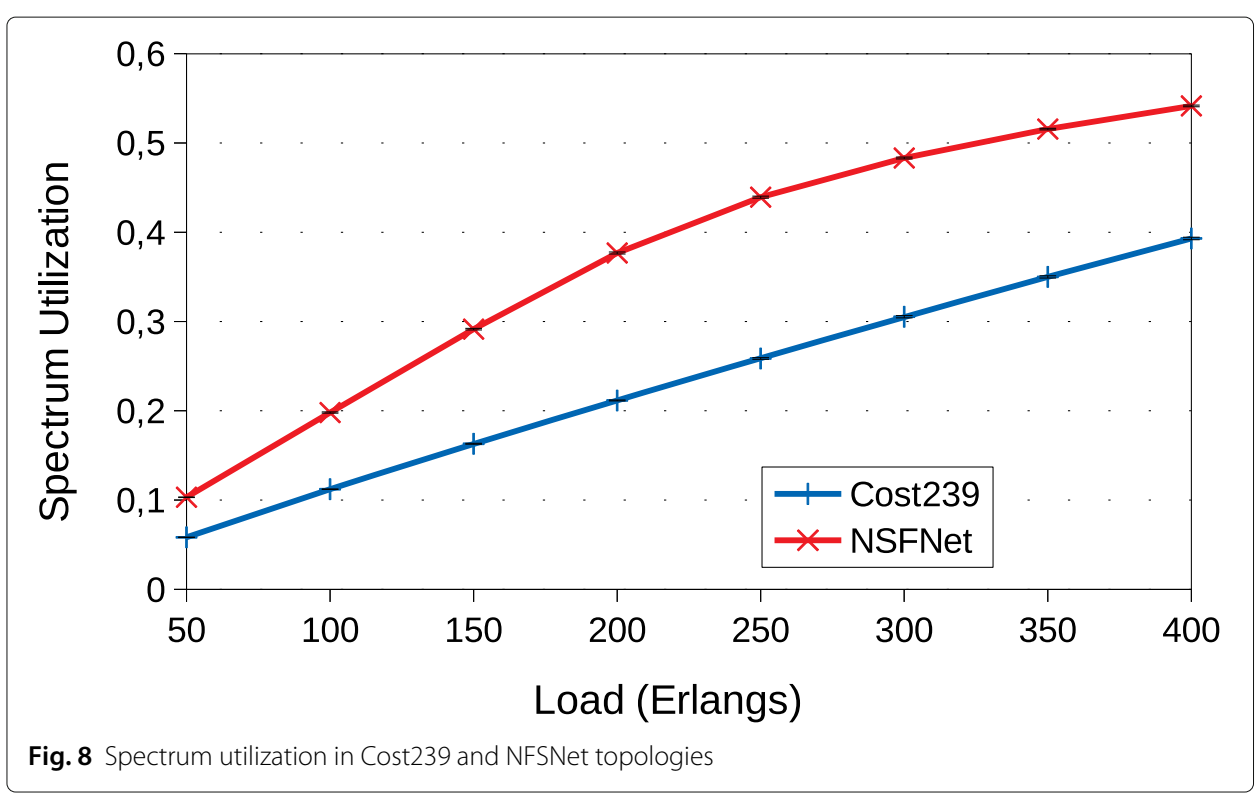

values as a function of the network load for Cost239 and NSFNet topologies (Fig. 10), considering the guard band equal to 8 slots which was the guard band value that maximized the network usage.

Observing the load point of 400 Erlangs in Fig. 8, the maximum usage achieved in Cost 239 and NSFNet topologies were $39 \%$ and $54 \%$, respectively. These maximum usage values were adjusted and divided by 7 to find the interval and increment for possible 8 guard band values. The guard band with value equal to 0 (zero) slots presented the worst performance in terms of blocking probability and is not considered by the GBUN algorithm. Table 1 presents the selection of the guard bands according to the spectrum usage for the Cost239 and NSFNet topologies.

The first step of the GBUN algorithm, when it is running, is to measure the usage of the network. With this information, the GBUN algorithm selects the appropriate guard band following Table 1. For example, if the spectrum usage measured by the GBUN algorithm in the Cost239 topology is equal to 0.19 at the time of arrival of a given circuit request, then, the selected guard band will be equal to 5 slots.

The other proposed guard band selection algorithm is the Guard Band by OSNR Margin (GBOM). Algorithm 1 presents the steps of GBOM. As input, GBOM has circuit information (circuit), a modulation format (modulation), a set of possible guard bands

Table 1 Selection of the guard bands according to the spectrum usage values (EspUti) of the network for Cost239 and NSFNet topologies

\begin{tabular}{lll}
\hline & Cost239 & NSFNet \\
\hline $\mathbf{G B}=\mathbf{1}$ & EspUti $\geq 0.35$ & EspUti $\geq 0.49$ \\
$\mathbf{G B}=\mathbf{2}$ & $0.35>$ EspUti $\geq 0.30$ & $0.49>$ EspUti $\geq 0.42$ \\
$\mathbf{G B}=\mathbf{3}$ & $0.30>$ EspUti $\geq 0.25$ & $0.42>$ EspUti $\geq 0.35$ \\
$\mathbf{G B}=\mathbf{4}$ & $0.25>$ EspUti $\geq 0.20$ & $0.35>$ EspUti $\geq 0.28$ \\
$\mathbf{G B}=\mathbf{5}$ & $0.20>$ EspUti $\geq 0.15$ & $0.28>$ EspUti $\geq 0.21$ \\
$\mathbf{G B}=\mathbf{6}$ & $0.15>$ EspUti $\geq 0.10$ & $0.21>$ EspUti $\geq 0.14$ \\
$\mathbf{G B}=\mathbf{7}$ & $0.10>$ EspUti $\geq 0.05$ & $0.14>$ EspUti $\geq 0.07$ \\
$\mathbf{G B}=\mathbf{8}$ & EspUti $<0.05$ & EspUti $<0.07$ \\
\hline
\end{tabular}


(guardBands) and a desired OSNR margin $(\sigma)$. The GBOM algorithm tests the possible guard bands in ascending order (line 3 ). For each selected guard band ( $g b$ ), GBOM applies the selected spectrum assignment algorithm considering the circuit information and the modulation format (line 4). If it is possible to allocate spectrum, the algorithm computes the QoT of the circuit (line 6). The physical layer model already presented is applied to determine the QoT of the circuit. If QoT is acceptable, the $\triangle O S N R$ is calculated (line 8). The OSNR threshold is the OSNR threshold of the modulation format. The guard band that reaches the $\triangle O S N R$ value greater than or equal to the desired OSNR margin is the guard band chosen to be used for the establishment of the new circuit.

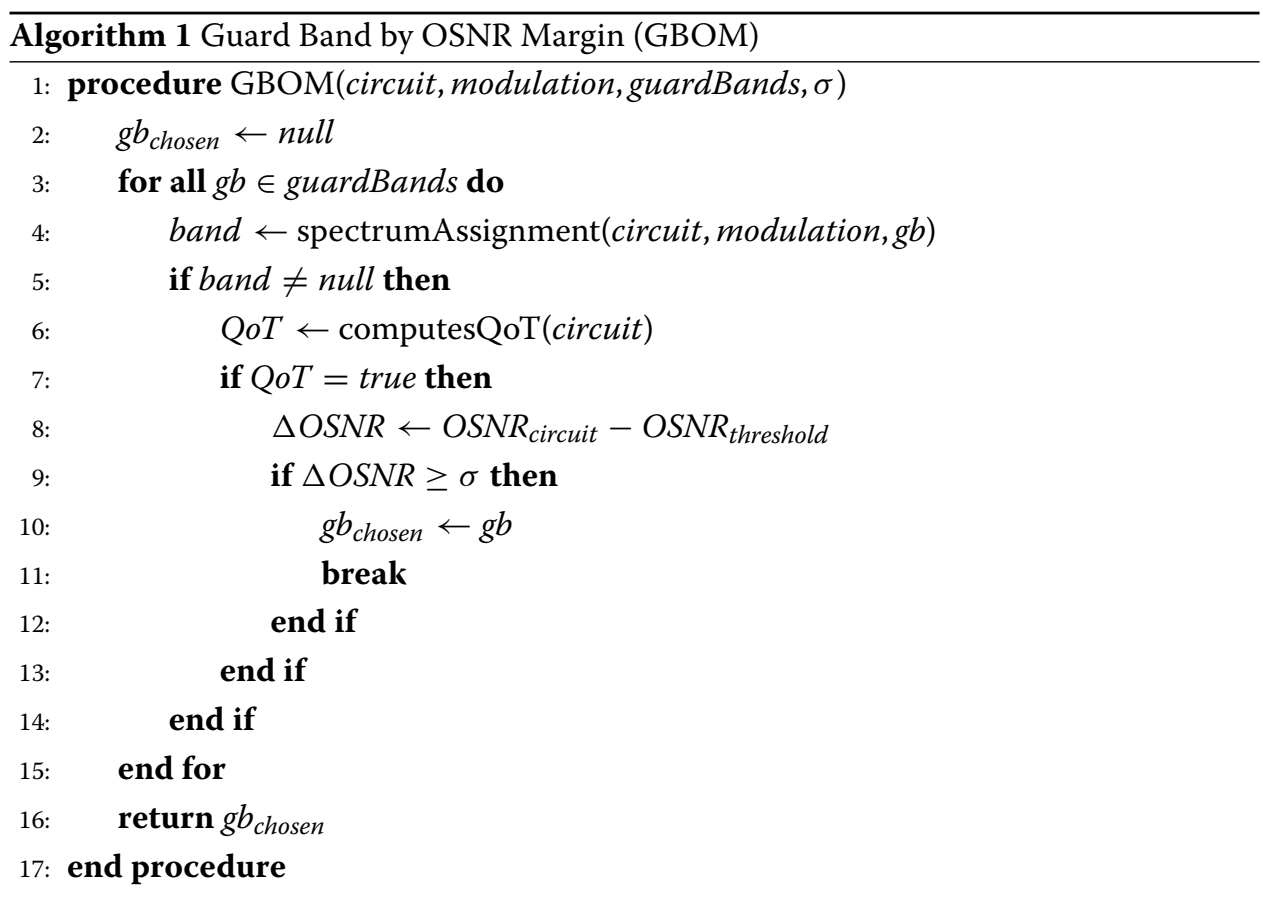

There might be more than one viable guard band for GBOM. The GBOM algorithm aims to choose the guard band to reduce physical layer impacts. For a particular circuit request(s,d) can at different times be attended using different guard bands. The choice of the guard band is dynamic, according to the state of the network. The GBOM algorithm analyzes the guard band possibilities in ascending slot size order (for example 1, 2, 3, 4, 5, 6,7 and 8 slots) to avoid excessive use of the optical spectrum. Following this search order, GBOM selects the guard band that guarantees the following requirements: i) spectrum availability (line 5); ii) an acceptable QoT (line 7) and iii) $\triangle O S N R$ greater than or equal to the chosen OSNR margin (line 9). GBOM search ends when it finds the first guard band that satisfies the three requirements described above (line 11).

The GBOM algorithm is not applied before the RMLSA algorithm. The GBOM algorithm is applied in conjunction with the modulation format selection algorithm. When the modulation format selection algorithm is testing the modulations, the GBOM algorithm select the guard band. That is, for each modulation that is tested, the GBOM algorithm is applied to select the most appropriate guard band. 
Experiments were conducted to select the value of $\sigma$ that minimizes the total blockage of the network. The sigma value was varied from 0.0 to 0.50 using steps of 0.05 . Figure 9 shows the bandwidth blocking probability obtained with each of the values of sigma for both topologies.

The result for $\sigma$ equal to 0.0 was removed from Fig. 9 due to the high blockage, and this enabled the other results to be seen more clearly. As seen in Fig. 9, the blockage was minimized when the value of $\sigma$ was equal to 0.10 , for both scenarios studied. The confidence interval is overlain with other values of $\sigma$, meaning that there is no significant difference between them. Consequently, the results presented in Section 5 for the GBOM algorithm used this value cited above $(\sigma=0.10)$.

The algorithm to solve the RMLSA problem adopted in this work was the Complete Sharing algorithm [18]. The Complete Sharing algorithm is an integrated RMLSA algorithm that has been proposed to deal with spectrum fragmentation. It uses routing with

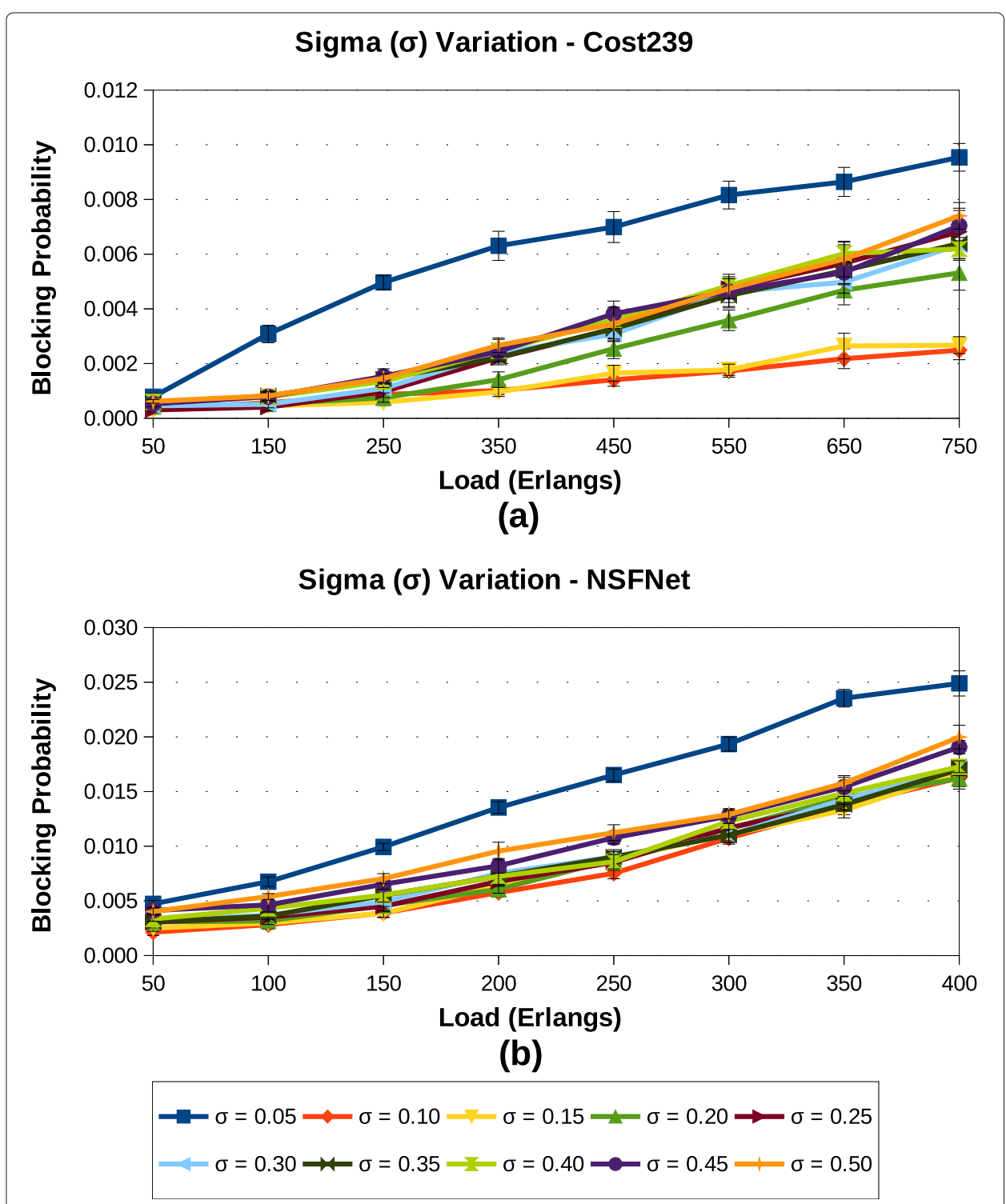

Fig. 9 Study of variation of the Sigma ( $\sigma$ ) for the topologies $\mathbf{a}$ Cost239 and $\mathbf{b}$ NSFNet 
$k$ candidate routes and selects the route that makes it possible to allocate the slot range nearest to the beginning of the spectrum. The Complete Sharing algorithm uses the First Fit for spectrum assignment. The modulation format selection algorithm by QoT is used [14].

\section{Performance evaluation}

The proposed algorithms GBUN and GBOM were compared with the adaptive algorithm AGBA [8] as well as with three fixed values for guard band found in the literature, which are: 0,1 and 2 slots. $G B=0$ corresponds to an attempt to use as much of the optical spectrum as possible. $\mathrm{GB}=1$ and $\mathrm{GB}=2$ are commonly used guard band values in the literature. The Complete Sharing algorithm was used to solve the RMLSA problem with $k$ equal to 3 . As a form of evaluation, the bandwidth blocking probability metrics were considered and the bandwidth blocking probability components were also analyzed. The components evaluated were: Fragmentation, QoTN (unacceptable QoT for a New circuit), QoTO (unacceptable QoT for Other circuits already active in the network) and AFS (Absence of Free Spectrum). Additionally, the algorithms were also evaluated considering the energy efficiency metrics.

The simulations were performed using the SNetS simulator (SLICE Network Simulator) [14]. Each simulation generated 100,000 requests for optical circuits with seven granularity levels: 100, 150, 200, 250, 300, 350 and 400 Gbps, with the proportions 7:6:5:4:3:2:1, respectively. The generation of requests follows the Poisson distribution with the traffic load evenly distributed among all the source and destination node. For each simulation ten replications were performed with different seeds of random variable generation. All results have a 95 percent confidence level. NSFNet (National Science Foundation Network) and Cost239 topologies were evaluated, as shown in Fig. 10, for the simulations of two different scenarios [19]. The modulation formats considered in this study were BPSK, 4QAM, 8QAM, 16QAM and 32QAM and their respective OSNR thresholds were $5.5 \mathrm{~dB}$, $8.5 \mathrm{~dB}, 12.5 \mathrm{~dB}, 15.1 \mathrm{~dB}$ and $18.1 \mathrm{~dB}$ [20]. All links in the network are bi-directional and have spectrum bandwidth divided into 320 frequency slots. A frequency slot has a bandwidth of $12.5 \mathrm{GHz}$. The amplifier gains are adjusted to compensate for device and fiber losses. Other parameters used in the simulations are listed in Table 2.

Figure 11 shows the bandwidth blocking probability obtained by AGBA, GBUN, GBOM and for fixed guard band values as a function of a network load for Cost239 and NSFNet topologies.

As seen in Fig. 11, the algorithm GBOM obtained a lower bandwidth blocking probability for both scenarios studied, considering all load points. Considering the 750 Erlangs load in the Cost239 topology, the algorithm GBOM presented a lower blocking probability of approximately $97.96 \%, 97.21 \%, 96.21 \%, 96.09 \%$ and $95.81 \%$ in relation to GB = $0, \mathrm{~GB}=1, \mathrm{~GB}=2, \mathrm{AGBA}$ and $\mathrm{GBUN}$, respectively. Considering the 400 Erlangs load in NSFNet topology, the algorithm GBOM showed a gain over the blocking probability of approximately $84.77 \%, 78.55 \%, 68.52 \%$ and $64.50 \%$ over $\mathrm{GB}=0, \mathrm{~GB}=1, \mathrm{~GB}=2$, AGBA and GBUN, respectively.

Tables 3 and 4 show the bandwidth blocking probability components obtained with the AGBA, GBUN and GBOM adaptive algorithms as well as for the fixed guard band values with a load of 750 Erlangs for the Cost239 topology and 400 Erlangs for the NSFNet topology. 


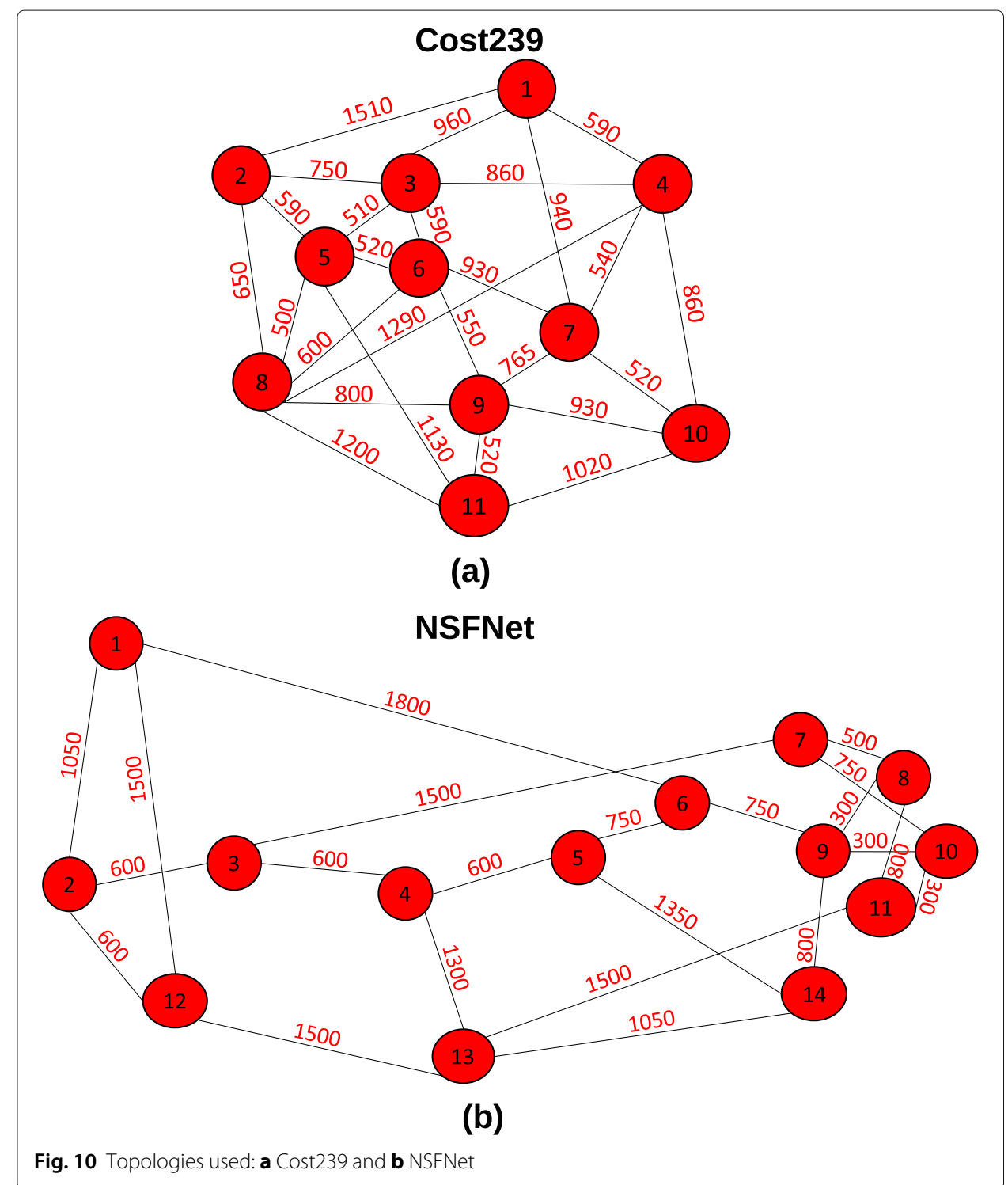

Tables 3 and 4 show that the QoTO component had the most impact on the overall blocking for all algorithms in both topologies. The use of the Complete Sharing algorithm contributes to the reduction of the blockages generated by the Fragmentation, AFS and QoTN components.

The GBUN had a better performance in relation to the fixed guard band values and the AGBA for most of the studied loads. This better performance of the GBUN algorithm

Table 2 Physical layer parameters used in simulations

\begin{tabular}{ll}
\hline Description & Value \\
\hline Signal power spectral density & $-23 \mathrm{dBm} / \mathrm{GHz}$ \\
Power attenuation $(\alpha)$ & $0.2 \mathrm{~dB} / \mathrm{km}$ \\
Fiber dispersion $(D)$ & $16 \mathrm{ps} /(\mathrm{nm} \cdot \mathrm{km})$ \\
Fiber non linearity coefficient $(\gamma)$ & $1.3(\mathrm{Wkm})^{-1}$ \\
Length of each span $\left(L_{s}\right)$ & $80 \mathrm{~km}$ \\
Noise figure of the amplifier $(N F)$ & $5 \mathrm{~dB}$ \\
\hline
\end{tabular}




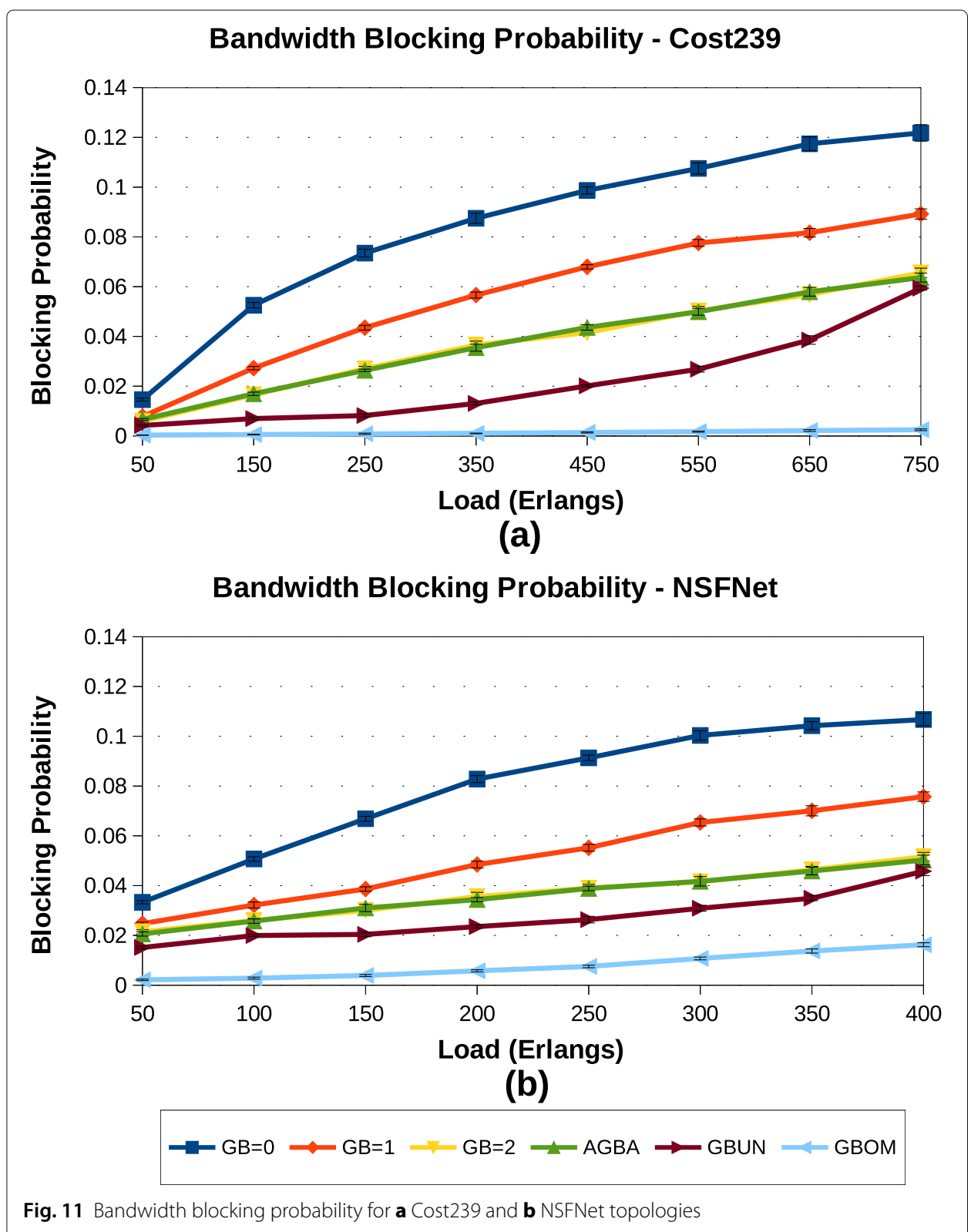

Table 3 Components of bandwidth blocking probability for Cost239 topology with a 750 Erlangs load

\begin{tabular}{lllll}
\hline & Fragmentation & QoTN & QoTO & AEL \\
\hline GB = 0 & $0 \%$ & $0 \%$ & $100 \%$ & $0 \%$ \\
GB = 1 & $0 \%$ & $0 \%$ & $100 \%$ & $0 \%$ \\
GB = 2 & $0 \%$ & $0 \%$ & $100 \%$ & $0 \%$ \\
AGBA & $0 \%$ & $0 \%$ & $100 \%$ & $0 \%$ \\
GBUN & $0 \%$ & $0 \%$ & $100 \%$ & $0 \%$ \\
GBOM & $1.01 \%$ & $0 \%$ & $98.99 \%$ & $0 \%$ \\
\hline
\end{tabular}


Table 4 Components of bandwidth blocking probability for NSFNet topology with a 400 Erlangs load

\begin{tabular}{lllll}
\hline & Fragmentation & QoTN & QoTO & AEL \\
\hline $\mathbf{G B}=\mathbf{0}$ & $0 \%$ & $0 \%$ & $100 \%$ & $0 \%$ \\
$\mathbf{G B}=\mathbf{1}$ & $0 \%$ & $0 \%$ & $100 \%$ & $0 \%$ \\
$\mathbf{G B}=\mathbf{2}$ & $0.32 \%$ & $0 \%$ & $99.66 \%$ & $0.02 \%$ \\
AGBA & $0.26 \%$ & $0 \%$ & $99.73 \%$ & $0 \%$ \\
GBUN & $0.71 \%$ & $0 \%$ & $99.25 \%$ & $0.03 \%$ \\
GBOM & $1.85 \%$ & $0.55 \%$ & $97.60 \%$ & $0 \%$ \\
\hline
\end{tabular}

is associated with its aim to use larger guard bands. This choice is based on the level of network usage, which indicates whether there are more or less slots available to increase or decrease the size of the guard band.

The better performance of GBOM in relation to the GBUN algorithm as well the other algorithms is also associated with the use of larger guard bands. The GBOM algorithm uses the same set of guard bands as GBUN. The main difference is that the GBOM algorithm uses more specific information from the circuits when selecting the appropriate guard bands.

Comparing the approaches as a function of the network topologies studied, it can be seen that the Cost239 topology was able to attend a higher traffic load than the NSFNet topology. This behavior is justified due to the Cost239 topology being more connected, and consequently has more alternative routes and resources to attend circuit requests. However, both topologies present the same performance order in terms of bandwidth blocking probability. It is important to highlight that the GBOM approach obtained a lower bandwidth blocking probability of 0.002 and 0.016 for the Cost239 and NSFNet topologies respectively, under the last load point.

Finally, the algorithms are also evaluated according to their energy efficiency. Energy efficiency is a metric that evaluates the total of bits transmitted in relation to the total energy consumed. The adopted energy consumption model $[21,22]$ considers the contribution of BVTs, BV-WXC and EDFAs amplifiers. The total energy consumption of the network is calculated by the sum of the total energy consumed by transponders, switches and EDFAs. Figure 12 shows the energy efficiency obtained by AGBA, GBUN, GBOM and for fixed guard band values as a function of a network load for Cost239 and NSFNet topologies, respectively.

Analyzing Fig. 12a, in general, the evaluated proposals do not present a significant difference in both topologies. Considering the 750 Erlangs load in the Cost239 topology, the algorithm GBOM presented a better energy efficiency of approximately $6.50 \%, 4.97 \%$, $1.98 \%, 2.85 \%$ and $2.88 \%$ in relation to $\mathrm{GB}=0, \mathrm{~GB}=1, \mathrm{~GB}=2$, AGBA and GBUN, respectively. The GBOM attends more circuits, so it transmits more bits and has a higher energy efficiency.

On the other hand, analyzing Fig. 12b, considering the 400 Erlangs load in the NSFNet topology, the algorithm GBOM presented a better energy efficiency of approximately $6.61 \%, 4.34 \%, 1.96 \%, 1.84 \%$ and $1.47 \%$ in relation to $\mathrm{GB}=0, \mathrm{~GB}=1, \mathrm{~GB}=2$, AGBA and GBUN, respectively. However, the confidence interval remains overlapping with $G B=2$, AGBA and GBUN, which means there is no significant difference in energy efficiency between these proposals. The same behavior can be observed at the other load points. 


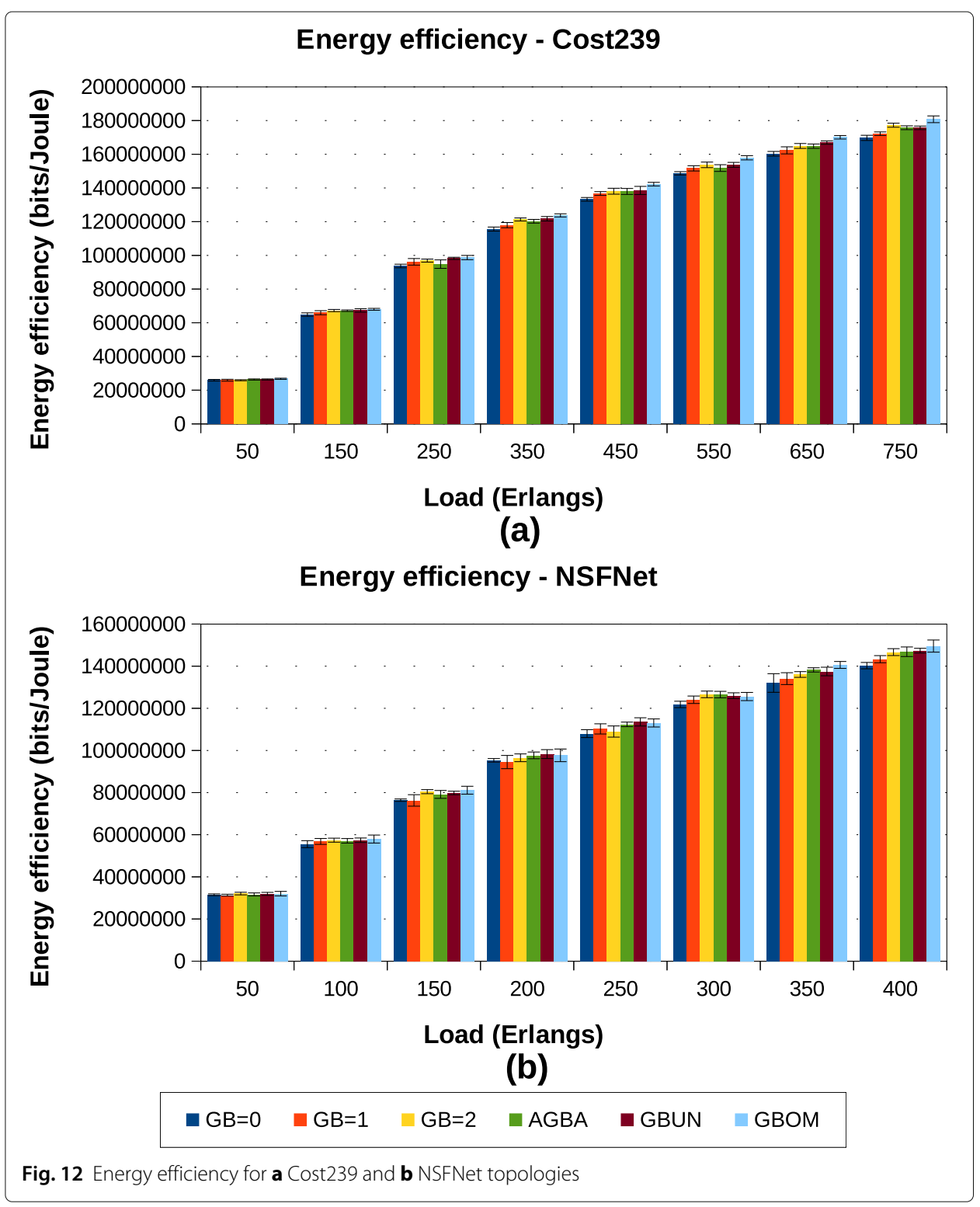

This shows that the guard band does not influence the energy efficiency of the network very much.

\section{Conclusion}

This article proposes two algorithms for adaptive guard band selection. The first algorithm, called GBUN, performs the selection of the guard band based on the state of spectrum use in the elastic optical networks. The choice of the guard band is made when the control plan receives a request to establish a circuit. The second algorithm, called GBOM, selects the guard band according to an OSNR margin. The GBOM algorithm is applied in conjunction with the modulation format selection algorithm. The objective of the proposed algorithms is to enable a better performance of the RMLSA algorithms with a guard band that adjusts to the current state of the network and thus reduce the probability of general blocking. 
A performance evaluation was carried out comparing the GBUN and GBOM algorithms with fixed guard band values commonly used in the literature and with the adaptive proposal AGBA. This evaluation was conducted using the Cost239 and NSFNet topologies.

The results of the performance evaluation study showed that the GBOM algorithm achieved the best bandwidth blocking probabilities compared to the AGBA algorithm, the use of fixed guard band and the GBUN proposal in both topologies. The GBOM algorithms achieves a reduction in the bandwidth blocking probability of at least $95.81 \%$ and $64.50 \%$ over GBUN, that achieved the second best performance, in Cost239 and NSFNet topologies, respectively.

As a future work we intend to evaluate the performance of the proposals with other RMLSA algorithms. We also intend to see how much an adaptive guard band selection improves the performance of physical layer aware RMLSA algorithms and to include other metrics in the performance evaluation such as fairness in the distribution of the block between communication pairs.

\begin{abstract}
Abbreviations
EONs: Elastic optical networks; OFDM: Orthogonal frequency division multiplexing; RMLSA: Routing, modulation level and spectrum assignment; IA: Impairment aware; BER: Bit error rate; GB: Guard band; QoT: Quality of transmission; GBUN: Guard band according to use of the network; GBOM: Guard band by OSNR margin; OSNR: Optical signal to noise ratio; BVT: Bandwidth variable transponders; BV-WXC: Bandwidth variable wavelength cross-connects; EDFA: Erbium doped fiber amplifiers; ASE: Amplified spontaneous emission; NLIs: Nonlinear impairments; SPM: Self-phase modulation; XPM: Cross-phase modulation; FWM: Four-wave mixing; PSD: Power spectral density; NF: Noise figure; QoTN: Inadequate QoT for the new circuit; QoTO: Inadequate QoT for others circuits already active in the network; QAM: Quadrature amplitude modulation; BPSK: Binary phase shift keying; Frag: Fragmentation; AFS: Absence of free spectrum; AGBA: Adaptive guard band assignment; NSFNet: National science foundation network; EspUti: Spectrum utilization/usage
\end{abstract}

\title{
Acknowledgements
}

The authors would like to thank the Departamento de Computação of the Universidade Federal do Piauí and the Centro de Informática of the Universidade Federal de Pernambuco that provided all support and incentive for the development of this work. This research was partially supported by CAPES and CNPq.

\section{Authors' contributions}

The authors NSM and ACF proposed and implemented the GBUM and GBOM algorithms respectively. The writing and review of the work was done equally by all authors. All author(s) read and approved the final manuscript.

\section{Availability of data and materials}

All the results obtained including information about how to replicate them in the simulator used can be found in this link: GitHub Repository

\section{Competing interests}

The authors declare that they have no competing interests.

\section{Author details}

${ }^{1}$ Departamento de Computação, Universidade Federal do Piauí, Teresina, PI, Brazil. ${ }^{2}$ Centro de Informática, Universidade Federal de Pernambuco, Recife, PE, Brazil.

Received: 23 March 2020 Accepted: 22 September 2020

Published online: 14 October 2020

\section{References}

1. Wu J, Ning Z, Guo L. Energy-efficient survivable grooming in software-defined elastic optical networks. IEEE Access. 2017;5:6454-63. https://doi.org/10.1109/ACCESS.2017.2674963.

2. Zhu P, Li J, Wu D, Chen Y, Tian Y, Wu Z, Ge D, Chen X, Chen Z, He Y. Software-defined elastic optical network node supporting spectrum defragmentation. IEEE/OSA J Opt Commun Netw. 2017;9(1):63-70. https://doi.org/10. 1364/JOCN.9.000A63.

3. Chatterjee BC, Sarma N, Oki E. Routing and spectrum allocation in elastic optical networks: A tutorial. IEEE Commun Surv Tutorials. 2015;17(3):1776-800. https://doi.org/10.1109/COMST.2015.2431731.

4. Christodoulopoulos K, Tomkos I, Varvarigos EA. Elastic bandwidth allocation in flexible OFDM-based optical networks. J Light Technol. 2011;29(9):1354-66. https://doi.org/10.1109/JLT.2011.2125777.

5. Beyranvand $H$, Salehi JA. A quality-of-transmission aware dynamic routing and spectrum assignment scheme for future elastic optical networks. J Light Technol. 2013;31(18):3043-54. https://doi.org/10.1109/JLT.2013.2278572.

6. Chen C, Ju M, Xiao S, Zhou F, Yang X. Minimizing total blocking by setting optimal guard band in nonlinear elastic optical networks. In: 2017 19th International Conference on Transparent Optical Networks (ICTON). IEEE; 2017. p. 1-4. https://doi.org/10.1109/ICTON.2017.8024956. 
7. Monteiro N, Soares A, Fontinele A, Santos IG, Campelo D, Oliveira A. Novo algoritmo para provisão de banda de guarda adaptativa em redes Ópticas elásticas. In: Anais do XXXVII Simpósio Brasileiro de Redes de Computadores e Sistemas Distribuídos. Porto Alegre, RS, Brasil: SBC; 2019. p. 307-20. https://doi.org/10.5753/sbrc.2019.7368 https:// sol.sbc.org.br/index.php/sbrc/article/view/7368.

8. Takeshita H, Noguchi H, Abe J, Fujisawa S, Tajima A. Adaptive guard-band assignment with adaptive spectral profile equalizer to improve spectral usage of impairment-aware elastic optical network. In: ECOC 2016; 42nd European Conference on Optical Communication. VDE; 2016. p. 1-3. https://ieeexplore.ieee.org/stamp/stamp.jsp? $\mathrm{tp}=$ \&arnumber $=7767550$ \&isnumber $=7766126$.

9. Jinno M, Takara H, Kozicki B, Tsukishima Y, Sone Y, Matsuoka S. Spectrum-efficient and scalable elastic optical path network: architecture, benefits, and enabling technologies. IEEE Commun Mag. 2009;47(11):66-73. https://doi.org/ 10.1109/MCOM.2009.5307468.

10. Zhao J, Wymeersch H, Agrell E. Nonlinear impairment aware resource allocation in elastic optical networks. In: 2015 Optical Fiber Communications Conference and Exhibition (OFC). OSA; 2015. p. 1-3. https://doi.org/10.1364/OFC. 2015.M2l.1.

11. Saradhi CV, Subramaniam S. Physical layer impairment aware routing (PLIAR) in WDM optical networks: Issues and challenges. Commun Surveys Tuts. 2009;11(4):109-30. https://doi.org/10.1109/SURV.2009.090407.

12. Johannisson P, Agrell E. Modeling of nonlinear signal distortion in fiber-optic networks. J Light Met. 2014;32(23): 4544-52. https://doi.org/10.1109/JLT.2014.2361357.

13. Yan L, Agrell E, Wymeersch $H$, Johannisson $P$, Di Taranto R, Brandt-Pearce M. Link-level resource allocation for flexible-grid nonlinear fiber-optic communication systems. IEEE Photon Technol Lett. 2015;27(12):1250-3. https:// doi.org/10.1109/LPT.2015.2415586.

14. Fontinele A, Santos I, Neto JN, Campelo DR, Soares A. An efficient IA-RMLSA algorithm for transparent elastic optical networks. Comput Netw. 2017;118:1-14. https://doi.org/10.1016/j.comnet.2017.03.003.

15. Costa LR, Drummond AC. New distance-adaptive modulation scheme for elastic optical networks. IEEE Commun Lett. 2017;21(2):282-5. https://doi.org/10.1109/LCOMM.2016.2624288.

16. Chen $X$, Zhong $Y$, Jukan A. Multipath routing in elastic optical networks with distance-adaptive modulation formats. In: 2013 IEEE International Conference on Communications (ICC). IEEE; 2013. p. 3915-20. https://doi.org/10. 1109/ICC.2013.6655169.

17. Sócrates-Dantas J, Careglio D, Perelló J, Silveira RM, Ruggiero WV, Solè-Pareta J. Challenges and requirements of a control plane for elastic optical networks. Comput Netw. 2014;72:156-71. https://doi.org/10.1016/j.comnet.2014.07. 007.

18. Wang R, Mukherjee B. Spectrum management in heterogeneous bandwidth optical networks. Opt Switch Netw. 2014;11 Part A:83-91. https://doi.org/10.1016/j.osn.2013.09.003.

19. Zhao Y, Hu L, Zhu R, Yu X, Wang X, Zhang J. Crosstalk-aware spectrum defragmentation based on spectrum compactness in space division multiplexing enabled elastic optical networks with multi-core fiber. IEEE Access. 2018;PP:1-1. https://doi.org/10.1109/ACCESS.2018.2795102.

20. Ives DJ, Bayvel P, Savory SJ. Physical layer transmitter and routing optimization to maximize the traffic throughput of a nonlinear optical mesh network. In: 2014 International Conference on Optical Network Design and Modeling. IEEE; 2014. p. 168-73. https://ieeexplore.ieee.org/stamp/stamp.jsp?tp=\&arnumber=6855789\&isnumber=6855750

21. Vizcaíno JL, Ye Y, Monroy IT. Energy efficiency analysis for flexible-grid ofdm-based optical networks. Comput Netw. 2012;56(10):2400-19. https://doi.org/10.1016/j.comnet.2012.03.012 Green communication networks.

22. Ju M, Zhou F, Xiao S, Zhu Z. Power-efficient protection with directedp-cycles for asymmetric traffic in elastic optical networks. J Light Technol. 2016;34(17):4053-65. https://doi.org/10.1109/JLT.2016.2590578.

\section{Publisher's Note}

Springer Nature remains neutral with regard to jurisdictional claims in published maps and institutional affiliations.

\section{Submit your manuscript to a SpringerOpen ${ }^{\circ}$ journal and benefit from:}

- Convenient online submission

- Rigorous peer review

- Open access: articles freely available online

- High visibility within the field

- Retaining the copyright to your article

Submit your next manuscript at $\boldsymbol{\triangleright}$ springeropen.com 